\title{
False Memory and Alzheimer's Disease Pathology in Patients with Amnestic Mild Cognitive Impairment: A Study with Amyloid PET
}

\author{
Eun-Ji Choi Bum Joon Kim Hyung-Ji Kim Miseon Kwon Noh Eul Han \\ Sun-Mi Lee Sungyang Jo Sunju Lee Jae-Hong Lee \\ Department of Neurology, Asan Medical Center, Seoul, South Korea
}

\begin{abstract}
Keywords
Alzheimer's disease pathology - Amnestic mild cognitive impairment · Amyloid positron emission tomography . False memory
\end{abstract}

\begin{abstract}
Introduction: False memory, observed as intrusion errors or false positives (FPs), is prevalent in patients with Alzheimer's disease, but has yet to be thoroughly investigated in patients with amnestic mild cognitive impairment (a-MCl) with Alzheimer's disease pathology (ADP). We analyzed false versus veridical memory in individuals with a- $\mathrm{MCl}$ and measured the utility of false memory for ADP discrimination. Methods: Patients with a-MCl who received neuropsychological testing and amyloid PET were included. Patients were categorized into "with" and "without ADP" groups according to PET results. Memory tests assessed veridical and false memory, and the verity of patient responses was analyzed. A logistic regression model was used to evaluate false memory efficiency in discriminating ADP, and the sensitivity and specificity at the optimal level were estimated using the receiver-operating characteristic curve. Results: Thirty-seven ADP and 46 non-ADP patients were enrolled. The ADP group made more FPs in the recognition tests, and their response verity was significantly lower in every delayed memory test. No group difference, however, was observed in the veridical
\end{abstract}

karger@karger.com www.karger.com/dee

Karger"

BOPEN ACCESS
(C) 2021 The Author(s)

Published by S. Karger AG, Basel

This is an Open Access article licensed under the Creative Commons Attribution-NonCommercial-4.0 International License (CC BY-NC) (http://www.karger.com/Services/OpenAccessLicense), applicable to the online version of the article only. Usage and distribution for commercial purposes requires written permission. memory. The logistic regression analysis demonstrated that as the FPs increased, the risk of ADP increased 1.31 and 1.36 times in the verbal and visual recognition tests, respectively. The discriminatory accuracy of the FPs was estimated "low" to "moderate" in the visual and verbal recognition, respectively, with an optimal cutoff above 2.5. Conclusion: Increased false memory was the only feature to discriminate ADP from non-ADP in individuals with a-MCl. Further studies regarding false memory and its mechanism are warranted.

(c) 2021 The Author(s)

Published by S. Karger AG, Basel

\section{Introduction}

Memory impairment has been widely recognized as a clinical presentation of prodromal Alzheimer's disease (AD). Biomarker studies have demonstrated that AD pathology (ADP) is more prevalent in individuals with amnestic mild cognitive impairment (a-MCI) [1]. However, a-MCI is caused by various etiologies, such as vascular lesion [2], Parkinson's disease [3], dementia with Lewy bodies [4], or frontotemporal dementia [5]. Besides, it is difficult to discriminate ADP within patients with a-MCI using the traditional approach of quantifying what the patient remembers in the given learning list [6]. Thus, there is a need for more thorough comprehension of the
Correspondence to:

Jae-Hong Lee, jhlee@ amc.seoul.kr 
distinctive amnestic features in individuals with MCI due to $\mathrm{AD}$.

Phenomenologically, in combination with loss of veridical memory, increased false memory has been indicated in patients with $\mathrm{AD}$ [7]. False memory describes the phenomena of incorrect memory that is mistaken as factual, derived from the imagination, contaminated by external stimulus or distorted by current knowledge, faith, or emotion. These memories are detected as intrusion errors in recall tests or false-positive responses to a recognition test. Despite their frequent occurrences, the literature on false memory in $\mathrm{AD}$ is very limited, with even less attention given to those at the MCI stage [8-11]. These days, a-MCI can be decisively separated into groups with ADP and non-ADP using amyloid PET as the National Institute on Aging and Alzheimer's Association (NIAAA) research framework guidelines encouraged [12].

We aimed to identify increased false memory in patients with a-MCI with ADP, who were at the predementia status, and to investigate whether false memory could discriminate ADP in individuals with a-MCI.

\section{Methods}

\section{Study Design and Participants}

A retrospective study was conducted in patients who visited the Department of Neurology at Asan Medical Center in Seoul, South Korea, with the chief complaint of cognitive impairment from July 2015 to January 2019. Patients with cognitive impairment who received neuropsychological evaluation and amyloid PET were screened. Of the total 100 patients at level 3 of the global deterioration scale (GDS), those with a-MCI were enrolled. a-MCI was defined by the results of verbal and visual memory tests, in which one or more subtest's $Z$ scores were below -1 by norms based on age and years of education in either immediate or delayed recall or recognition tests. Patients were excluded if they had other medical conditions that may lead to cognitive decline, assessed by serological tests including a complete blood count, chemistry battery, vitamin $B_{12}$, folate, syphilis, HIV serology, and thyroid function tests. Patients with psychiatric disorders or a history of drug abuse and those with structural brain lesions confirmed by brain imaging studies were also excluded.

Patients were divided into groups depending on the presence of ADP, measured by fluorine-18 $\left[{ }^{18} \mathrm{~F}\right]$-florbetaben PET. The memory test results were then compared between the 2 groups.

$\left[{ }^{18} \mathrm{~F}\right]$-Florbetaben PET

$\left[{ }^{18} \mathrm{~F}\right]$-florbetaben PET was performed at Asan Medical Center for $\beta$-amyloid plaque confirmation, a diagnostic feature of $\mathrm{AD}$. This technique is known as one of the latest imaging study measures to aid the early differentiation of AD based on its high sensitivity and specificity [13]. Discovery 690/710/690 Elite (GE Healthcare, Milwaukee, WI, USA) was used to obtain the images, and scanning was conducted 90-110 min after intravenous adminis- tration of $300 \mathrm{MBq}$ florbetaben. The images were regarded as amyloid negatives when categorized as brain amyloid plaque load 1 and as positives when it was 2 or 3 , based on the predefined regional cortical tracer binding and the brain amyloid plaque load system.

\section{Assessment of Memory Impairment}

Using the Seoul Neuropsychological Screening Battery 2nd Edition [14], trained psychologists conducted neuropsychological evaluations. The immediate and delayed recall tests and recognition tests from the Seoul Verbal Learning Test (SVLT) and the Rey-Osterrieth Complex Figure Test (RCFT) were used in this study.

In the SVLT immediate recall test, 12 learning items were verbally presented; the list comprised 3 semantic categories (flowers, kitchen utensils, and stationery) with 4 items per category. Three learning trials were conducted, and the patient's score was based on the sum of their correct responses (CRs). The delayed recall test was conducted $20 \mathrm{~min}$ later and the same scoring method applied. Following this, the recognition test was conducted: 12 learning and 12 nonlearning items were presented, with half of the nonlearning list semantically related to the learning list (flowers, utensils, stationery); the patient had to decide whether the items belonged to the previous learning list.

The patients drew what they could remember in the RCFT immediate recall test, which took place directly after the RCFT copy task. The delayed recall test was then conducted within 20 min of the immediate recall test. Patient outcomes were scored based on the standard protocol. The RCFT recognition test followed the delayed recall test: 24 items were presented, composed of 12 correct and incorrect elements of the RCFT figure; the patient decided whether each component belonged to the previously presented figure.

The SVLT and RCFT recall test scores were used to evaluate veridical memory directly. The number of true positive responses (TPs) and recognition discriminability (the patient's ability to discriminate veridical items from lures) were also used.

To analyze false memory in recall tests, intrusion errors (IEs) in the SVLT immediate and delayed recall tests were counted, additionally. Also, the new variable of "the verity of recall test responses $\left(\mathrm{V}_{\text {Recall }}\right)$ " was computed to numerically present the verity of the responses: the lower the verity of the responses due to intrusion errors, the lower the $\mathrm{V}_{\text {Recall }}$ (Table 1). Additionally, false-positive responses (FPs) in the SVLT and RCFT recognition tests were assessed. In particular, "false positive responses to the semantically related items (FP-Rs)" and "false positives to the semantically unrelated items (FP-URs)" in the SVLT recognition test were analyzed. The new variables "verity of positive responses in recognition test $\left(\mathrm{V}_{\mathrm{P}}\right)$ " and "verity of negative responses in recognition test $\left(V_{N}\right)$ " were then computed to present the verity of positive and negative responses numerically. A lower $V_{P}$ indicated a lower verity of positive responses, whereas a lower verity of negative responses gave a lower $\mathrm{V}_{\mathrm{N}}$ (Table 1$)$.

\section{Statistical Analysis}

All statistical analyses were conducted using SPSS PASW Statistics 18.0 (SPSS Inc., Chicago, IL, USA), and the level of statistical significance was set at $p \leq 0.05$. The Kolmogorov-Smirnov test was performed to examine the assumption of abnormality before group comparisons were conducted according to the results of the 
Table 1. Variables used in the analysis

Recall test

SVLT CR, the number of correct responses

$0-36$ in the immediate recall test, $0-12$ in the delayed recall test

$\mathrm{IE}$, the number of intrusion errors

$\mathrm{V}_{\text {Recall }}$, the verity of responses in the recall test, $(\mathrm{CR}-\mathrm{IE}) /(\mathrm{CR}+\mathrm{IE}),-1-1^{\mathrm{a}, \mathrm{b}}$

RCFT The scores of drawing, 0-36

Recognition test

SVLT

Percent recognition discriminability, $100 \times(\mathrm{TP}+\mathrm{TN}) / 24,0-100$

$\mathrm{TP}$, the number of true positives, $0-12$

$\mathrm{FP}$, the number of false positives, $0-12$

FP-R, the number of false positives to the semantically related items, 0-6

FP-UR, the number of false positives to the semantically unrelated items, 0-6

$\mathrm{V}_{\mathrm{P}}$, the verity of positives, $(\mathrm{TP}-\mathrm{FP}) /(\mathrm{TP}+\mathrm{FP}),-1-1^{\mathrm{a}, \mathrm{c}}$

$\mathrm{V}_{\mathrm{N}}$, the verity of negatives, $\left.(\mathrm{TN}-\mathrm{FN}) / \mathrm{TN}+\mathrm{FN}\right),-1-1^{\mathrm{a}, \mathrm{d}}$

RCFT

Percent recognition discriminability, $100 \times(\mathrm{TP}+\mathrm{TN}) / 24,0-100$

$\mathrm{TP}$, the number of true positives, $0-12$

FP, the number of false positives, $0-12$

$\mathrm{V}_{\mathrm{P}}$, the verity of positives, $(\mathrm{TP}-\mathrm{FP}) /(\mathrm{TP}+\mathrm{FP}),-1-1^{\mathrm{a}, \mathrm{c}}$

$\mathrm{V}_{\mathrm{N}}$, the verity of negatives, $(\mathrm{TN}-\mathrm{FN}) /(\mathrm{TN}+\mathrm{FN}),-1-1^{\mathrm{a}, \mathrm{d}}$

\footnotetext{
a In each calculation of the $\mathrm{V}_{\text {Recall }}, \mathrm{V}_{\mathrm{P}}$, and $\mathrm{V}_{\mathrm{N}}$, in case of " $\mathrm{CR}=\mathrm{IE}=0$," "TP $=\mathrm{FP}=0$," and "TN $=\mathrm{FN}=0$," each variable was replaced by 1 , to comply with the nonparametric estimation procedure. ${ }^{\mathrm{b}}$ When the CR is fixed, the more the IEs, the lower the $\mathrm{V}_{\text {Recall }}$. When $\mathrm{IE}=0, \mathrm{~V}_{\text {Recall }}=1$, when $\mathrm{CR}=\mathrm{IE}, \mathrm{V}_{\text {Recall }}=0$, and when $\mathrm{CR}=0$, $\mathrm{V}_{\text {Recall }}=-1$. $^{\mathrm{c}}$ When the TP is fixed, the more the FPs, the lower the $\mathrm{V}_{\mathrm{P}}$. When $\mathrm{FP}=0, \mathrm{~V}_{\mathrm{P}}=1$, when TP=FP, $\mathrm{V}_{\mathrm{P}}=0$, and when $\mathrm{TP}=0, \mathrm{~V}_{\mathrm{P}}=-1 .{ }^{\mathrm{d}}$ When the TN is fixed, the more the FNs, the lower the $\mathrm{V}_{\mathrm{N}}$. When $\mathrm{FN}=0$, $\mathrm{V}_{\mathrm{N}}=1$, when $\mathrm{TN}=\mathrm{FN}, \mathrm{V}_{\mathrm{N}}=0$, and when $\mathrm{TN}=0, \mathrm{~V}_{\mathrm{N}}=-1$.
}

Table 2. Group comparisons of characteristics and the level of global cognitive functions depending on ADP in patients with a-MCI

\begin{tabular}{lllr}
\hline & ADP $(n=37)$ & Non-ADP $(n=46)$ & $p$ value \\
\hline Mean age (SD), years & $71.1(7.6)$ & $71.0(8.3)$ & 0.944 \\
Females, $n$ (\%) & $20(54.1)$ & $27(58.7)$ & 0.824 \\
Median years of education (IQR) & $12.0(7.0$ to 16.0$)$ & $12.0(9.0$ to 16.0$)$ & 0.845 \\
ApoE $\varepsilon 4$ carrier, $n(\%)^{\mathrm{a}}$ & $16(69.6)$ & $7(17.9)$ & $<0.001$ \\
Median K-MMSE (IQR) & $27.0(24.0$ to 28.0$)$ & $26.5(24.0$ to 29.0$)$ & 0.592 \\
Median CDR-SOB (IQR) & $1.5(1.0$ to 2.5$)$ & $1.5(1.0$ to 2.0$)$ & 0.297 \\
$Z$ score of SNSB-II cognitive domain score & & & 0.102 \\
$\quad$ Median attention (IQR) & $-0.30(-0.50$ to 0.39$)$ & $-0.49(-0.86$ to 0.07$)$ & 0.227 \\
$\quad$ Median language (IQR) & $-0.46(-1.58$ to 0.25$)$ & $-0.13(-1.33$ to 0.46$)$ & 0.397 \\
$\quad$ Median visuospatial (IQR) & $-0.15(-1.27$ to 0.45$)$ & $-0.55(-1.44$ to 0.35$)$ & 0.037 \\
$\quad$ Median memory (IQR) & $-1.99(-2.96$ to -1.53$)$ & $-1.54(-2.09$ to -1.04$)$ & 0.387 \\
$\quad$ Median frontal/executive (IQR) & $-1.00(-2.08$ to 0.07$)$ & $-0.79(-1.70$ to 0.10$)$ & \\
\hline
\end{tabular}

ADP, Alzheimer's disease pathology; a-MCI, amnestic mild cognitive impairment; ApoE ع4, apolipoprotein E ع4; CDR-SOB, Clinical Dementia Rating Scale - Sum of Box; IQR, interquartile range; K-MMSE, Korean version of Mini-Mental Status Examination; SD, standard deviation; SNSB-II, Seoul Neuropsychological Screening Battery-II. ${ }^{a}$ An ApoE genotype test was carried out in a total of 23 patients with ADP and 39 with nonADP. ${ }^{b}$ Every patient's CDR score is the same at 0.5 . 
Table 3. Group comparisons of memory test results in patients with a-MCI depending on ADP

\begin{tabular}{|c|c|c|c|}
\hline & $\operatorname{ADP}(n=37)$ & Non-ADP $(n=46)$ & $p$ value \\
\hline \multicolumn{4}{|l|}{ SVLT immediate recall } \\
\hline Median CR (IQR) & $15.0(11.0$ to 17.0$)$ & $15.0(11.0$ to 17.0$)$ & 0.593 \\
\hline Median IE (IQR) & $2.0(1.0$ to 5.0$)$ & $2.0(1.0$ to 3.25$)$ & 0.091 \\
\hline Cases with IE occurrence ${ }^{a, \%}$ & $31(83.8)$ & $36(78.3)$ & 0.586 \\
\hline Median $V_{\text {Recall }}(\mathrm{IQR})$ & $0.7(0.5$ to 0.9$)$ & $0.8(0.7$ to 0.9$)$ & 0.377 \\
\hline \multicolumn{4}{|l|}{ SVLT delayed recall } \\
\hline Median CR (IQR) & $2.0(0.0$ to 3.5$)$ & $2.5(1.8$ to 4.0$)$ & 0.073 \\
\hline Median IE (IQR) & $1.0(0.0$ to 2.0$)$ & $1.0(0.0$ to 3.0$)$ & 0.799 \\
\hline Cases with IE occurrence $^{a}$ & $27(73.0)$ & $31(67.4)$ & 0.636 \\
\hline Median $V_{\text {Recall }}(\mathrm{IQR})$ & $0.0(-0.3$ to 0.3$)$ & $0.2(0.0$ to 0.7$)$ & 0.030 \\
\hline \multicolumn{4}{|l|}{ SVLT recognition } \\
\hline Median recognition discriminability, \% (IQR) & $70.8(66.7$ to 79.2$)$ & $79.2(70.8$ to 84.4$)$ & 0.041 \\
\hline Median TP (IQR) & $9.0(7.0$ to 11.0$)$ & $9.0(7.0$ to 11.0$)$ & 0.608 \\
\hline Median FP (IQR) & $3.0(2.0$ to 5.0$)$ & $2.0(1.0$ to 3.0$)$ & $<0.001$ \\
\hline FP-R, median (IQR) & $2.0(2.0$ to 4.0$)$ & $1.0(1.0$ to 3.0$)$ & $0.014^{\mathrm{b}}$ \\
\hline Median FP-UR (IQR) & $0.0(0.0$ to 1.5$)$ & $0.0(0.0$ to 0.0$)$ & 0.044 \\
\hline Median $V_{P}(I Q R)$ & $0.5(0.3$ to 0.7$)$ & $0.7(0.5$ to 0.8$)$ & 0.001 \\
\hline Median $\mathrm{V}_{\mathrm{N}}(\mathrm{IQR})$ & $0.5(0.3$ to 0.8$)$ & $0.5(0.3$ to 0.7$)$ & 0.938 \\
\hline \multicolumn{4}{|l|}{ RCFT immediate recall } \\
\hline Median raw score (IQR) & $6.0(4.0$ to 11.0$)$ & $9.0(4.9$ to 12.6$)$ & 0.258 \\
\hline \multicolumn{4}{|l|}{ RCFT delayed recall } \\
\hline Median raw score (IQR) & $6.0(3.8$ to 9.0$)$ & 8.5 (4.9 to 12.0$)$ & 0.073 \\
\hline \multicolumn{4}{|l|}{ RCFT recognition } \\
\hline Median recognition discriminability, \% (IQR) & $70.8(62.5$ to 83.3$)$ & $79.2(70.8$ to 83.3$)$ & 0.041 \\
\hline Median TP (IQR) & $9.0(7.0$ to 11.0$)$ & $9.0(7.0$ to 10.0$)$ & 0.601 \\
\hline Median FP (IQR) & $3.0(2.0$ to 5.0$)$ & $2.0(1.0$ to 3.0$)$ & 0.004 \\
\hline Median $V_{P}(I Q R)$ & $0.4(0.3$ to 0.6$)$ & $0.6(0.5$ to 0.8$)$ & 0.003 \\
\hline Median $\mathrm{V}_{\mathrm{N}}(\mathrm{IQR})$ & $0.5(0.2$ to 0.8$)$ & 0.5 (0.3 to 0.7$)$ & 0.752 \\
\hline \multicolumn{4}{|c|}{$\begin{array}{l}\text { ADP, Alzheimer's disease pathology; a-MCI, amnestic mild cognitive impairment; FP, false positives; IE, } \\
\text { intrusion error; IQR, interquartile range; RCFT, Rey-Osterrieth Complex Figure Test; SVLT, Seoul Verbal } \\
\text { Learning Test; TP, true positives; } \mathrm{V}_{\mathrm{N}} \text {, verity of negative responses; } \mathrm{V}_{\mathrm{P}} \text {, verity of positive responses; } \mathrm{V}_{\text {Recall }} \text {, verity } \\
\text { of responses. }{ }^{\text {a }} \text { The number of cases that exhibited intrusion errors in the test. }{ }^{b} \text { Due to Bonferroni correction for } \\
\text { multiple testing, the } p \text { value was set at } 0.025 \text {. }\end{array}$} \\
\hline
\end{tabular}

amyloid PET analysis. The parametric independent sample $t$ test and the nonparametric Mann-Whitney $U$ test were then carried out. Cross-tabulation analysis was performed to compare categorical variables such as the sex ratio, the ratio of apolipoprotein $\mathrm{E}$ (ApoE) $\varepsilon 4$ carriers as a risk factor for $\mathrm{AD}$, and IE's prevalence in the SLVT recall tests.

Logistic regression analysis of the backward stepwise elimination using the likelihood ratio was performed using memory test results to explore the usefulness in estimating ADP. The variables that were entered into the model were as follows: demographics such as age, sex, and years of education; and memory test variables such as the CRs and IEs in the SVLT immediate and delayed recall tests, the score from RCFT immediate and delayed recall tests, and the TPs and FPs in SVLT and RCFT recognition tests. The sensitivity and specificity of the tests' variables for differentiating in $\mathrm{AD}$ were analyzed at the optimal level on the receiver-operating characteristic curve.

\section{Results}

A total of 201 individuals who underwent both amyloid PET and cognitive battery were enrolled. After the GDS screening, those at GDS level $2(n=20)$, whose memory deterioration was regarded as subjective, and those at GDS levels 4 and 5 ( $n=64$, and 17 , respectively), whose cognitive deterioration level was moderate or severe, were excluded. Of the 100 patients with MCI at GDS level 3, 17 patients with non-a-MCI were excluded, leaving a total of 83 patients with a-MCI enrolled.

Among the 83 participants, 36 were male and 47 were female, and their mean age was 71.0 years (SD 7.9). The median years of education were 12.0 (IQR 9.0-16.0), and the median Korean version of the Mini-Mental State Examination was 27.0 (IQR 24.0-28.0). 
Fig. 1. The result of qualitative analysis on memory tests. Test performance above -1 $Z$ score was regarded as in the normal range. ADP, Alzheimer's disease pathology; FP, false positive; RCFT, Rey-Osterrieth Complex Figure Test; SVLT, Seoul Verbal Learning Test; TP, true positive; $(+)$, the ADP group; (-), the non-ADP group. ${ }^{*} p<$ 0.05. ${ }^{* *} p<0.01$.

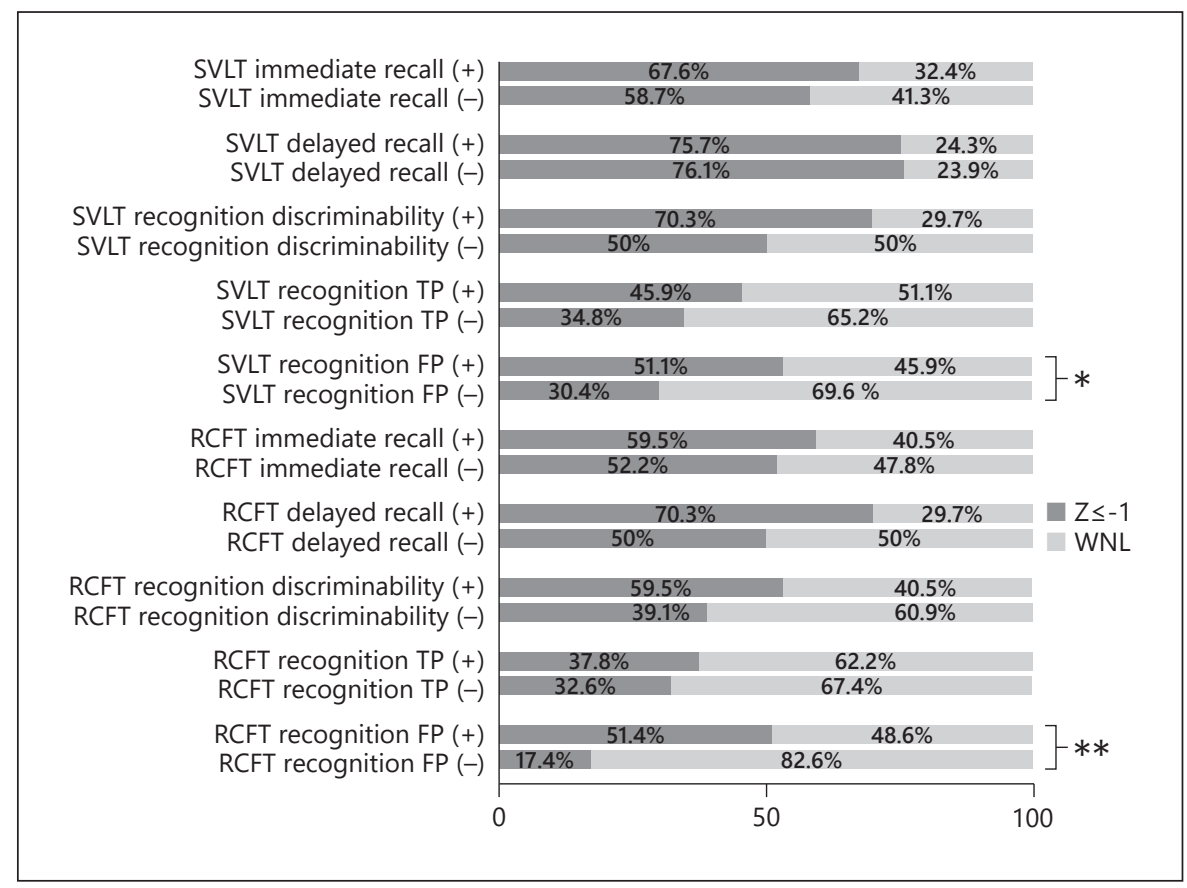

\section{Demographics and Clinical Characteristics}

Of the total 83 patients, 37 patients had ADP and 46 did not. Table 2 summarizes the demographics, clinical characteristics, and the level of global cognitive function. The group comparison analysis showed that the risk of carrying ApoE $\varepsilon 4$ genes was higher in the ADP group than in the non-ADP group (odds ratio $=10.4, \chi^{2}=16.52$, $p<0.001)$. However, there were no significant differences in sex ratio, age, and years of education between the groups.

The neuropsychological examination indicated that the cognitive domain score of memory was lower in the ADP group $(t[81]=2.26, p<0.05)$; however, there were no group differences in the other cognitive domain scores in attention, language, visuospatial, and frontal/executive domains, as well as the Korean version of the Mini-Mental State Examination and Clinical Dementia Rating scale scores. The Clinical Dementia Rating score of both groups was 0.5 .

\section{Group Comparison of Memory Test Results}

The memory tests' results depending on the groups are described in Table 3. The analysis of immediate recall tests showed no significant differences. However, the ADP group's $V_{\text {Recall }}$ in the delayed recall test was significantly lower than that of the non-ADP group ( $U=618.5$, $p<0.05)$. Also, the median of the delayed recall test in this group was 0 , indicating that at least half of the ADP group patients tended to make as many IEs as they did CRs.

The results of the recognition test indicated that the ADP group made more FPs in the SVLT and RCFT (SVLT, $\mathrm{U}=488.5, p<0.001$; RCFT, $\mathrm{U}=629.5, p<0.05$ ). In the SVLT, both ADP and non-ADP groups tended to make more FP-Rs than FP-URs. Above all, the FP-R level in the ADP group was higher even after Bonferroni correction for multiple testing comparisons $(\mathrm{U}=587.5, p<$ $0.025)$. The ADP group had lower $V_{P}$, in the SVLT and RCFT, compared with the non-ADP group (SVLT, $\mathrm{U}=$ 487.5, $p<0.001$; RCFT, $U=527.0, p<0.01$ ). However, neither the TPs nor the $V_{N}$ exhibited any differences in the SVLT and the RCFT recognition tests.

As for the FPs in the SVLT recognition test, the proportion (\%) of the cases outside the normal range was $54.1 \%$ in the ADP group and $18.7 \%$ in the non-ADP group $\left(\chi^{2}=4.73, p<0.05\right)$; in the RCFT recognition test, the proportion (\%) of cases outside the normal range was $51.4 \%$ in the ADP group and $17.4 \%$ in the non-ADP group $\left(\chi^{2}=10.78, p<0.01\right)$. There was no difference in the other group comparisons (Fig. 1).

\section{Predictors for the Presence of ADP}

ADP discrimination results, using the memory test results obtained from the logistic regression analysis of the backward stepwise elimination and likelihood ratio, are 
Table 4. Estimates for ADP and 95\% confidence intervals for the odds ratio in the final logistic regression model

\begin{tabular}{lllll}
\hline & Odds ratio & Lower confidence limit & Upper confidence limit & $p$ value \\
\hline FP in SVLT recognition test & 1.307 & 1.014 & 1.684 & $<0.05$ \\
FP in RCFT recognition test & 1.357 & 1.038 & 1.774 & $<0.05$ \\
\hline
\end{tabular}

ADP, Alzheimer's disease pathology; FP, false positive; RCFT, Rey-Osterrieth Complex Figure Test; SVLT, Seoul Verbal Learning Test.

Table 5. The sensitivity and specificity of indices from memory tests in differentiating ADP in patients with aMCI: at the optimal level estimated using ROC curve

\begin{tabular}{lllllll}
\hline & Cutoff & AUC & $\begin{array}{l}\text { Standard } \\
\text { error }\end{array}$ & $\begin{array}{l}\text { Asymptotic } \\
\text { significance }\end{array}$ & Sensitivity, \% & Specificity, \% \\
\hline FP in SVLT recognition test & 2.5 & 0.713 & 0.057 & 0.001 & 54.1 & 73.9 \\
FP in RCFT recognition test & 2.5 & 0.683 & 0.060 & 0.004 & 70.3 & 67.4 \\
\hline
\end{tabular}

ADP, Alzheimer's disease pathology; a-MCI, amnestic mild cognitive impairment; AUC, area under the curve; FP, false positive; RCFT, Rey-Osterrieth Complex Figure Test; ROC, receiver-operating characteristic; SVLT, Seoul Verbal Learning Test.

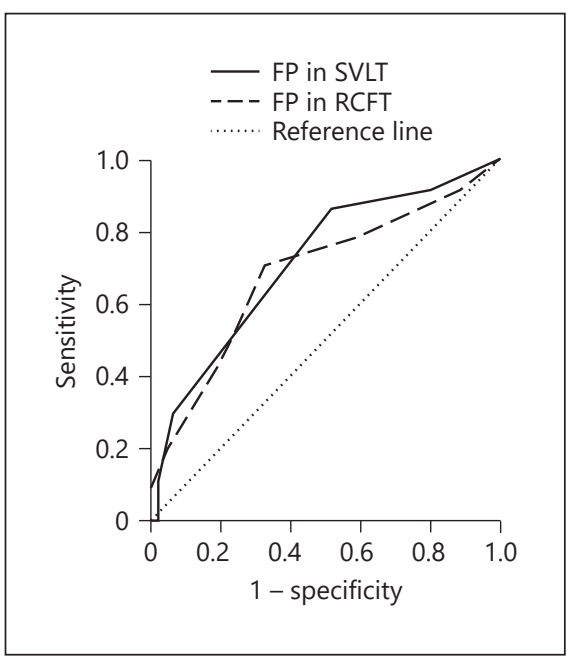

Fig. 2. The receiver-operating characteristic curve of the number of FPs in the SVLT and RCFT recognition tests. FPs, false positives; RCFT, Rey-Osterrieth Complex Figure Test; SVLT, Seoul Verbal Learning Test.

shown in Table 4 . The outcome variables from the memory tests used in the analysis were CRs and IEs in the SVLT immediate and delayed recall tests, and TPs and FPs in the SVLT recognition tests. The outcome variables collected from the RCFT were scores from the immediate and delayed recall tests, and TPs and FPs in the recognition tests. Demographic and clinical factors, including variables of sex, age, and years of education, were analyzed.

The final model included the FPs in the SVLT and RCFT recognition tests. The estimated model using those 2 variables was significant at $p<0.01$, with its regression equation explaining $21.3 \%$ of the phenomenon. According to the model, the FP's odds ratio increased 1.307 and 1.357 times in the SVLT and RCFT recognition tests, respectively.

\section{Diagnostic Accuracy of False Memory for ADP}

Table 5 describes the sensitivity and the specificity of the memory test indices at the receiver-operating characteristic curve's optimal level. The indices that proved applicable to discriminating ADP in patients with a-MCI were the FPs from the SVLT and RCFT recognition tests (Fig. 2). The FPs in the SVLT recognition test had a "moderate level" of diagnostic accuracy [15] with a sensitivity of $54.1 \%$ and a specificity of $73.9 \%$ at the optimal cutoff of above 2.5. The FPs in the RCFT recognition test showed "low" accuracy [15] with a sensitivity of $70.3 \%$ and a specificity of $67.4 \%$ at this optimal cutoff of above 2.5 . No other variables exhibited significant diagnostic accuracy for ADP. 


\section{Discussion}

Patients with a-MCI were categorized into "with" ADP and "without" ADP groups according to the results of the amyloid PET analysis. Memory test results were compared, focusing on false memory concerning IEs in the recall tests and FPs in the recognition tests. In brief, the ADP group did not perform as well in memory tests as the non-ADP group. The group difference was prominent in the level of false memory, but CRs failed to discriminate the groups. In the verbal delayed recall test, the ADP group had a significantly lower verity of responses, suggesting that their responses were more biased towards intrusion errors than the non-ADP group. A similar pattern was observed in the recognition test, with more FP responses in the ADP group than in the non-ADP group. Results suggest that the ADP group's positive responses were more likely to be false both in the verbal and visual tests.

This increased false memory production in patients with ADP is consistent with AD research previously published in the literature. For instance, in a study conducted in patients with various causes of dementia, the group with lower cerebrospinal fluid $A \beta_{1-42}$ level (indicative of ADP) displayed a higher frequency of FPs in the picture recognition test [10]. Additionally, in a retrospective study with dementia with Lewy bodies and $A D$ verified by autopsy [8], patients with AD generated more IEs in delayed cued recall tests and had a higher frequency of FPs. They also showed a tendency for positive response bias in the CVLT recognition tests compared with patients with dementia with Lewy bodies. Similar results have also been observed in comparative studies between patients with progressive supranuclear palsy [9] and Huntington's disease [11] compared with patients with $\mathrm{AD}$, which noted that the $\mathrm{AD}$ group displayed an increased IE rate and a higher frequency of FPs.

Little is known about the mechanism underlying false memory in individuals with ADP, but there seems to be a distinctive FP response pattern that can be observed in repetitive learning conditions. For example, a previous study found that TP responses gradually increased in every group across the repetitive learning trials; however, the number of FPs gradually decreased in the control group, whereas such a pattern was not evident in those with medial temporal lobe damage and even steadily increased in the group with Korsakoff syndrome [16]. Another study found that the number of TPs increased gradually in the $\mathrm{AD}$, young, and older control groups during the repetitive learning trials; in contrast, the increased FP tendency was observed only in patients with $\mathrm{AD}$ [17].
In this study, there was a difference between the a-MCI groups with and without ADP with respect to false memory production, but not in the sum of CRs. Their different veridical and false memory patterns may suggest that separate mechanisms exist in correctly affirming the exact the learned items and in rejecting the false lures. A previous study highlighted that patients with $\mathrm{AD}$ exhibited the distinctive false memory pattern, likely because they failed to reject semantic gist information [17]. This suggestion was based on the fuzzy-trace theory that proved two distinctive memory systems through experiments: verbatim and gist traces [18]. The verbatim traces refer to representations in which the surface forms of information are encoded as they are; on the other hand, the gist traces are conceptual and are interpreted at the varied levels of meaning, relations, or patterns (e.g., after reading "spaniel," various levels of concepts can be stored such as "hound," "cattle," or "pet") [18]. Both systems contribute to remembrance. However, while the verbatim retrieval enables re-experience of the original information through vivid recollection and helps to inhibit false memory, gist retrieval may provoke vulnerability to confusion of similar information.

We noted that FPs tended to occur more in the semantically related items than in the unrelated ones across both groups. This seemingly supports the fuzzy-trace theory, which suggests that gist retrieval may trigger false memory vulnerability in items that share meanings. Notably, the ADP group made more FPs in the semantically related items, suggesting that a-MCI patients with ADP were more vulnerable to semantically related false memory than a-MCI patients without ADP. In light of the fuzzy-trace theory, increased FPs in the ADP group could be associated with a defective inhibition process of false memory, whether it is due to having a poorer verbatim memory or to their relatively strengthened gist system.

According to the complementary learning system model [19], encoding and identifying specific details rely more on the activation of the hippocampus, whereas the medial temporal lobe cortex is more involved in integrating common nature to the current knowledge and discerning the familiarity based on the contrasts between learning and lure information. Considering this, increased false memory in patients with ADP in this study may therefore be related to hippocampal dysfunction or relatively elevated medial temporal lobe cortex function. It is plausible that our results may be associated with these structures given that they are known to present early pathological changes in AD [20-22]. Further studies will be required to identify the effects that these brain components have on increasing false memory in ADP. 
Lastly, we estimated ADP using false memory within patients with a-MCI. As a result of the logistic regression analysis, the FPs in the recognition tests were indicators of ADP's risk among memory indices. The discriminatory accuracy of FPs in the recognition tests was evaluated as "low to moderate," at the optimal cutoff of above 2.5 (AUC of the SVLT recognition test $=0.713$; AUC of the RCFT recognition test $=0.683$ ). With such discriminatory powers, it seems that the false memory measures are insufficient to be directly applied to the clinical scene. However, increased false memory still holds value for further study: first, we strictly enrolled patients with GDS level 3 a-MCI, and at this mildly dysfunctional level, the veridical memory level did not effectively differentiate the ADP group from the non-ADP groups with a-MCI; moreover, our $\beta$-amyloid positivity prediction using false memory was somewhat comparable with the result from 130 patients with a-MCI using hippocampal volume measures (AUC $=0.723$ ) [6].

The significance of this study is as follows. We recruited patients with a-MCI from the clinical scene and diagnosed their ADP using amyloid PET as NIA-AA research framework encouraged [12]. Additionally, we analyzed the veridical and false memory separately according to the presence of ADP and focused on the verity of memory in patients with ADP, which had thus far been somewhat overlooked. As a result, we found that the level of false memory increased in MCI patients with ADP as in $\mathrm{AD}$ patients in the literature, suggesting that the false memory may serve as an aid to the detection of prodromal AD. Memory distortion can defame even the value of correct remembrance and considerably affect the lives of individuals with ADP. Research on false memory will provide a deeper understanding of memory impairment associated with $\mathrm{AD}$ and can contribute to guidance on cognitive training at a more practical level.

There are also several limitations to this study. Memory test profiles between patients with and without ADP were compared but were based solely on amyloid PET analysis. Thus, the nature of non-ADP is unknown. Since a-MCI patients without ADP can have various pathologies, further segregation of non-ADP according to its pathology and lesion topography is warranted. More extensive neuroimaging and biomarker utilization may also be required. Second, we failed to exclude other concomitant pathologies in the ADP group. It is well known that ADP in elderly patients often overlaps with vascular disease [23] or dementia with Lewy bodies [24], so it will be necessary to compare memory profiles in the future with mixed conditions more strictly controlled.
Investigating the underlying mechanism of false memory will be important in future research. A further study incorporating neuroimaging and biomarkers is also warranted to identify brain areas or circuits relevant to veridical and false memory in patients with ADP, and to track changes in the false memory pattern with ADP progression.

\section{Statement of Ethics}

The present study protocol was reviewed and approved by the Institutional Review Board of Asan Medical Center (approval No. 2020-1737). The board waived the requirement of informed consent.

\section{Conflict of Interest Statement}

The authors have no potential conflicts of interest to disclose.

\section{Funding Sources}

This study was supported by a grant of the Korea Health Technology R\&D Project through the Korea Health Industry Development Institute (KHIDI), funded by the Ministry of Health and Welfare, Republic of Korea (grant No. HI18C2383).

\section{Author Contributions}

E.-J.C. and N.E.H.: conceptualization. E.-J.C.: data curation, formal analysis. J.-H.L.: funding acquisition. E.-J.C., H.-J.K., S.L., S.J., S.-M.L., and M.K.: investigation. E.-J.C., H.-J.K., and B.J.K.: methodology. E.-J.C.: writing - original draft. B.J.K., N.E.H, and J.-H.L.: writing - review and editing.

References

1 Jansen WJ, Ossenkoppele R, Knol DL, Tijms BM, Scheltens P, Verhey FR, et al.; Amyloid Biomarker Study Group. Prevalence of cerebral amyloid pathology in persons without dementia: a meta-analysis. JAMA. 2015 May;313(19):1924-38.

2 Zanetti M, Ballabio C, Abbate C, Cutaia C, Vergani C, Bergamaschini L. Mild cognitive impairment subtypes and vascular dementia in community-dwelling elderly people: a 3-year follow-up study. J Am Geriatr Soc. 2006 Apr;54(4):580-6.

3 Chiaravalloti ND, Ibarretxe-Bilbao N, DeLuca J, Rusu O, Pena J, García-Gorostiaga I, et al. The source of the memory impairment in Parkinson's disease: acquisition versus retrieval. Mov Disord. 2014 May;29(6):765-71. 
4 Donaghy PC, Taylor JP, O'Brien JT, Barnett $\mathrm{N}$, Olsen K, Colloby SJ, et al. Neuropsychiatric symptoms and cognitive profile in mild cognitive impairment with Lewy bodies. Psychol Med. 2018 Oct;48(14):2384-90.

5 de Mendonça A, Ribeiro F, Guerreiro M, Garcia C. Frontotemporal mild cognitive impairment. J Alzheimers Dis. 2004 Feb;6(1):1-9.

6 Alves L, Cardoso S, Silva D, Mendes T, Marôco J, Nogueira J, et al. Neuropsychological profile of amyloid-positive versus amyloidnegative amnestic mild cognitive impairment. J Neuropsychol. 2020 Jun;e12218.

7 El Haj M, Colombel F, Kapogiannis D, Gallouj K. False memory in Alzheimer's disease. Behav Neurol. 2020 Feb;2020:5284504.

8 Hamilton JM, Salmon DP, Galasko D, Delis DC, Hansen LA, Masliah E, et al. A comparison of episodic memory deficits in neuropathologically confirmed dementia with Lewy bodies and Alzheimer's disease. J Int Neuropsychol Soc. 2004 Sep;10(5):689-97.

9 Pillon B, Deweer B, Michon A, Malapani C, Agid Y, Dubois B. Are explicit memory disorders of progressive supranuclear palsy related to damage to striatofrontal circuits? Comparison with Alzheimer's, Parkinson's, and Huntington's diseases. Neurology. 1994 Jul;44 (7):1264-70.

10 Hildebrandt $\mathrm{H}$, Haldenwanger A, Eling $\mathrm{P}$ False recognition correlates with amyloid$\beta(1-42)$ but not with total tau in cerebrospinal fluid of patients with dementia and mild cognitive impairment. J Alzheimers Dis. 2009; 16(1):157-65.
11 Jacobs D, Salmon DP, Tröster AI, Butters N. Intrusion errors in the figural memory of patients with Alzheimer's and Huntington's disease. Arch Clin Neuropsychol. 1990;5(1):4957.

12 Jack CR Jr, Bennett DA, Blennow K, Carrillo MC, Dunn B, Haeberlein SB, et al.; Contributors. NIA-AA Research Framework: toward a biological definition of Alzheimer's disease. Alzheimers Dement. 2018 Apr;14(4):535-62.

13 Barthel H, Gertz HJ, Dresel S, Peters O, Bartenstein P, Buerger K, et al.; Florbetaben Study Group. Cerebral amyloid- $\beta$ PET with florbetaben $(18 \mathrm{~F})$ in patients with Alzheimer's disease and healthy controls: a multicentre phase 2 diagnostic study. Lancet Neurol. 2011 May;10(5):424-35.

14 Kang Y. Jahng, S. Na D. Seoul Neuropsychological Screening Battery. 2nd ed. Incheon: Human Brain Research \& Consulting Co; 2012.

15 Grzybowski M, Younger JG. Statistical methodology. III. Receiver operating characteristic (ROC) curves. Acad Emerg Med. 1997 Aug;4(8):818-26.

16 Schacter DL, Verfaellie M, Anes MD, Racine C. When true recognition suppresses false recognition: evidence from amnesic patients. J Cogn Neurosci. 1998 Nov;10(6):668-79.

17 Budson AE, Daffner KR, Desikan R, Schacter $\mathrm{DL}$. When false recognition is unopposed by true recognition: gist-based memory distortion in Alzheimer's disease. Neuropsychology. 2000 Apr;14(2):277-87.
18 Brainerd CJ, Reyna VF. Fuzzy-trace theory and false memory. Curr Dir Psychol Sci. 2002;11(5):164-9.

19 O'Reilly RC, Norman KA. Hippocampal and neocortical contributions to memory: advances in the complementary learning systems framework. Trends Cogn Sci. 2002 Dec;6(12):505-10

20 Price JL, Ko AI, Wade MJ, Tsou SK, McKeel DW, Morris JC. Neuron number in the entorhinal cortex and CA1 in preclinical Alzheimer disease. Arch Neurol. 2001 Sep;58(9):1395402.

21 Deweer B, Lehéricy S, Pillon B, Baulac M, Chiras J, Marsault C, et al. Memory disorders in probable Alzheimer's disease: the role of hippocampal atrophy as shown with MRI. J Neurol Neurosurg Psychiatry. 1995 May; 58(5):590-7.

22 Dickerson BC, Salat DH, Bates JF, Atiya M, Killiany RJ, Greve DN, et al. Medial temporal lobe function and structure in mild cognitive impairment. Ann Neurol. 2004 Jul;56(1):2735.

23 Chui HC, Ramirez-Gomez L. Clinical and imaging features of mixed Alzheimer and vascular pathologies. Alzheimers Res Ther. 2015 Feb;7(1):21.

24 Constantinides VC, Paraskevas GP, Boufidou F, Bourbouli M, Paraskevas PG, Stefanis L, et al. Cerebrospinal fluid amyloid beta and tau proteins in atypical Parkinsonism: a review. Neuroimmunol Neuroinflamm. 2020;2020: $120-31$. 